\title{
Molecular genetic techniques and oligonucleotides for mycoplasma identification - a review
}

\author{
Markhabat Kairova, Damegul Rakhimzhanova \\ Kazakh AgroTechnical University named after S. Seifullin (KATU), Nur-Sultan, Republic of Kazakhstan
}

Received May 15, 2020

Accepted December 21, 2020

\begin{abstract}
Control of distribution of mycoplasmal infections in cattle herds is essential in the majority of countries world-wide. Various PCR procedures are available to detect mycoplasmas in cell cultures and bovine mycoplasma in different types of samples. We reviewed some common PCR techniques and specific primers targeted to different bacterial genetic regions of mycoplasma. Several researchers used the same PCR approach and Mycoplasma spp. as a target but their results could not be compared because different primer pairs were used. These methods and primers were first developed to identify mycoplasma species that contaminate animal cell cultures, and then were used by other researchers to differentiate mycoplasmas as a cow infecting agent. Our analysis of the specificity of these primer pairs to nucleotide sequences of five Mycoplasma spp. showed that oligonucleotides have less specificity to them. Numerous commercially available PCR kits are applicable to find mycoplasma contamination in cell cultures and fewer of them can be used in veterinary diagnostics. Although serological and culture techniques are still used, it is necessary to develop a new multiplex PCR technique with a more specific primer set especially in agrarian countries.
\end{abstract}

PCR, species-specific primers, sequencing, nested PCR, real-time PCR, cow disease

It is known that in industrial cattle breeding, the use of a basic method of reproduction such as artificial insemination does not always guarantee a high fertility rate of cows due to the possibility of semen contamination. Breeding efficiency depends largely on timely prevention and treatment of the diseases of reproductive organs that cause infertility, lowering the number of calves born. Poor uterine conditions due to obstetrical and genital diseases (such as retention of placenta, endometritis, subinvolution of the uterus etc.) cause alterations in the fertilization process and can also lead to cattle infertility (Marusich 2017).

In recent years, farms in Kazakhstan have suffered major economic losses due to the wide distribution of non-contagious animal diseases. Among the opportunistic microorganisms associated with non-contagious diseases, mycoplasmas are of great importance, and in synergy with the viral and bacterial microbiota, they can increase the severity of various diseases in the cattle, including respiratory diseases, diseases of the reproductive organs, urogenital tract, arthritis, otitis, and others.

Inflammation of the cattle mammary gland is often caused by various microorganisms, including streptococci and staphylococci (Zhumanov et al. 2015). However, in 1961, mycoplasmas were isolated in cows with severe mastitis in the USA. It has been identified that Mycoplasma bovis is the most important mycoplasmal pathogen in farm animals worldwide. This species of mycoplasma is the causative agent of mastitis and arthritis in adult cows and is also reportedly associated with cases of abortions (Hotzel et al. 1993). The second most common mycoplasma in cattle is $M$. californicum, associated with mastitis in adults, as well as arthritis and pneumonia in calves. The species of M. bovigenitalium is isolated from the cow's reproductive tract and is associated with vulvovaginitis and

Address for correspondence:

Damegul Rakhimzhanova

Kazakh AgroTechnical University

named after S. Seifullin (KATU)

Nur-Sultan, Zhenis avenue, 62

Republic of Kazakhstan 010011

Phone: +77018247711

E-mail: rahimzhanova2011@mail.ru

http://actavet.vfu.cz/ 
infertility, as well as postnatal diseases such as dystocia and endometritis (Parker et al. 2017).

At present, more than 100 mycoplasma species have been described, many of which are pathogenic to humans and animals (cows, sheep, pigs, birds) whereas other mycoplasmas infect plants and insects. Studies have shown that not all species of mycoplasma can cause disease, animals are often carriers of the pathogen and the infection process can be asymptomatic for a long time. The percentage of cows affected by mastitis is known to be $25 \%$ annually, while the disease can be progressing in a latent form in more than $50 \%$ of animals (Adilbekova et al. 2015).

The traditional method of mycoplasma detection is their cultivation on highly enriched isolation media such as Spiroplasma medium SP-4, Difco pleuropneumonia like organism broth (PPLO), Friis medium, Hayflick's agar medium containing 10-20\% of horse serum (Friis 1975; Nakagaw a etal. 1992; Tang etal.2000). However, the microbiological method of cultivation has a number of limitations, including their slow growth within 7-30 days, as well as difficulties in differentiating Mycoplasma species and its discrimination from non-pathogenic Acholeplasma bacteria with similar colonial morphology (Hotzel et al. 1993). Because of the highly contagious nature of the pathogen and the effects that bovine mycoplasma may have on the entire herd, it is important to rapidly obtain the final results to minimize the spread of infection. Methods of serological analysis can be used to screen $M$. bovis antibodies or antigens in clinically diseased animals, so early detection of infection is not possible in this case ( $\mathrm{Sachse}$ et al. 1993). Diagnosis of genital infections associated with mycoplasmas through PCR analysis is a much quicker, sensitive and specific method, and has several advantages, especially if some strains of mycoplasma are non-culturable. In this study, we investigated the main molecular genetic techniques and primer pairs used to detect and identify mycoplasmal pathogens, including infectious agents that can be involved in the decrease of cattle fertility.

\section{PCR techniques for mycoplasma detection}

Developed in the 1990s, the traditional PCR method used for mycoplasma identification is based on the amplification of conserved bacterial 16S rRNA gene. The use of available NCBI GenBank and EMBL nucleotide databases with alignment of 16S rRNA sequences made it possible to select genus-specific MGSO-GPO-1 primers to detect species of the Mycoplasma genus, as well as other mycoplasmas such as Ureaplasma spp., Spiroplasma spp. and Acholeplasma spp. (Van Kuppeveld et al. 1992). Although PCR with universal $\mathrm{pH}-\mathrm{pA}$ pair primers and further partial $16 \mathrm{~S}$ rRNA sequencing related to the gold standard method are often used for identification of mycoplasma species. To identify 60 strains from 27 different mycoplasma species and subspecies, Stakenborg (2005) and co-authors used the ARDRA method, based on the digestion of the amplified fragments of the conserved $16 \mathrm{~S}$ rRNA locus. However, this method requires a large number of restriction endonucleases, including $A l u \mathrm{I}\left(\mathrm{AG}^{\wedge} \mathrm{CT}\right), B f a \mathrm{I}\left(\mathrm{C}^{\wedge} \mathrm{TAG}\right), H p y \mathrm{FIOVI}\left(\mathrm{GCNNNNN}{ }^{\wedge} N \mathrm{NGC}^{\circ}\right.$ (Stakenborg et al. 2005).

Like most prokaryotic organisms, mycoplasmas have 1-2 operons containing ribosomal RNA (rRNA) genes. It has been established that in all mycoplasmas, the DNA sequence of the spacer region located between the $16 \mathrm{~S}$ rRNA and $23 \mathrm{~S}$ rRNA genes is rich in A and T nucleotides, and also does not contain genes encoding transport RNA (tRNA). In a study of $16 \mathrm{~S}-23 \mathrm{~S}$ rRNA spacer region in Acholeplasma laylawii and using primers developed for the identification of lactobacilli, it was found that unlike mycoplasma, acholeplasma contains two loci of tRNA (Nakagawa et al. 1992). Comparative study of the sequences of 16S-23S rRNA spacer region was carried out for phylogenic analysis of mycoplasma species belonging to cluster M. mycoides (Harasawa et al. 2000). In that work, PCR 


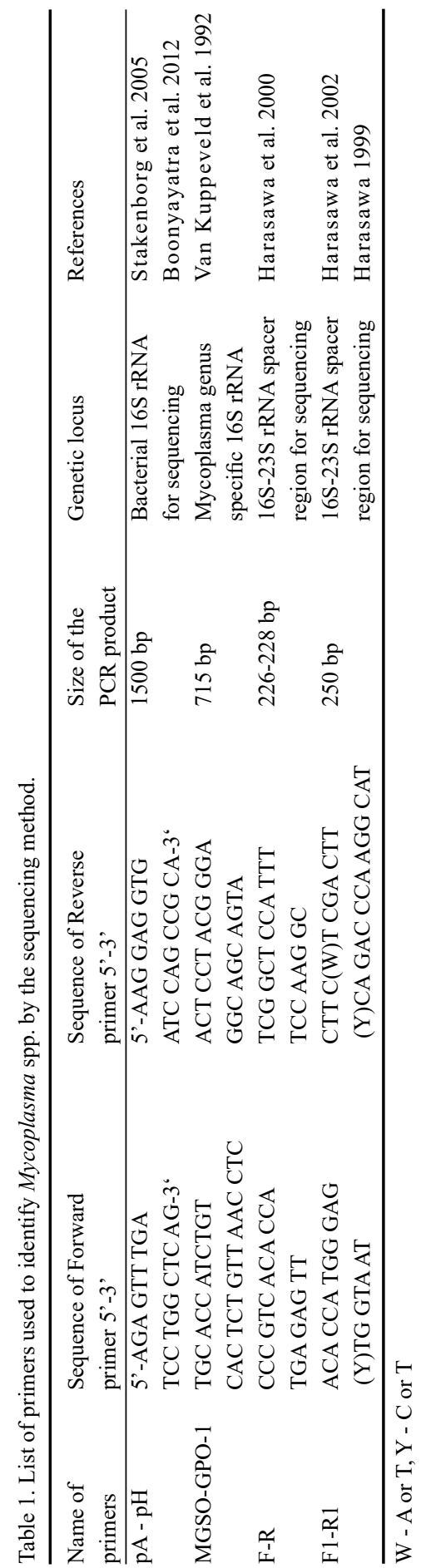

was carried out with F-R primers, and then products of DNA amplification were sequenced. However, in other studies, as well as in studying the genetic relationships of genetically related mycoplasmas and the species of $M$. haemomuris, which was previously identified as Haemobartonella muris, researchers already provided sequences of other F1-R1 primers (Harasawa 1999; Harasawa et al. 2002). Table 1 shows the nucleotide sequences of primers used in genus-specific PCR and in the sequencing genetic loci of mycoplasmas.

The PCR amplification with primers specific to the conserved region of the $16 \mathrm{~S}$ rRNA gene must be accompanied by using primers peculiar to each bacterial species. For the detection and discrimination between different mycoplasma species as contaminants in cell cultures, Harasawa et al. (1993) have developed a nested PCR method with two pairs of primers necessary to amplify a heterogeneous $16 \mathrm{~S}-23 \mathrm{~S}$ rRNA spacer region. This work is based on PCR with flanking MCGpF11-R23-R1 and internal R162-MCGpR21 primers, which were derived from the known nucleotide sequence of the conserved region of rRNA operon of mycoplasma (Nakagawa et al. 1992). As a result of PCR, the primer set generated one specific DNA fragment of 236-365 base pairs (bp) with each of the 8 mycoplasma species of cell culture contaminants, while $A$. laylawii gave a specific pattern with two discrete bands of 430 and 223 bp in length. Due to the fact that some closely related species of mycoplasma give a cross-reaction during serological analysis, as well as for the differentiation of strains within a single species, the RFLP analysis of PCR products is often used. Thus, the identification of 9 species and strains of mycoplasma was done by digestion of DNA fragments using VspI, HindIII, HincII, ClaI, PvuII, HaeIII enzymes (Harasawa et al. 1993). The sequences of oligonucleotides used in these studies are shown in Table 2.

The sensitivity for the PCR detecting A. laidlawii was increased to the level of $20-180 \mathrm{CFU} / \mathrm{ml}$ by incorporating three additional primers specifically selected from $16 \mathrm{~S}$ and $23 \mathrm{~S}$ rRNA region of the species (Tang et al. 2000). In the process of mycoplasma detection, Tang et al. (2000) used the same primer set of MCGpF11-R23-R1 and R16-2-MCGpR21 indicated as F1-R1 and F2-R2, but in this work with reference to Nakagawa (1992), we can see another reverse primer R1 with sequence of 5'-CTT C(A/T)T CGA CTT (C/T/)CA GAC CCA AGG CAT-3' that is recommended by Harasawa (1996) and Harasawa 


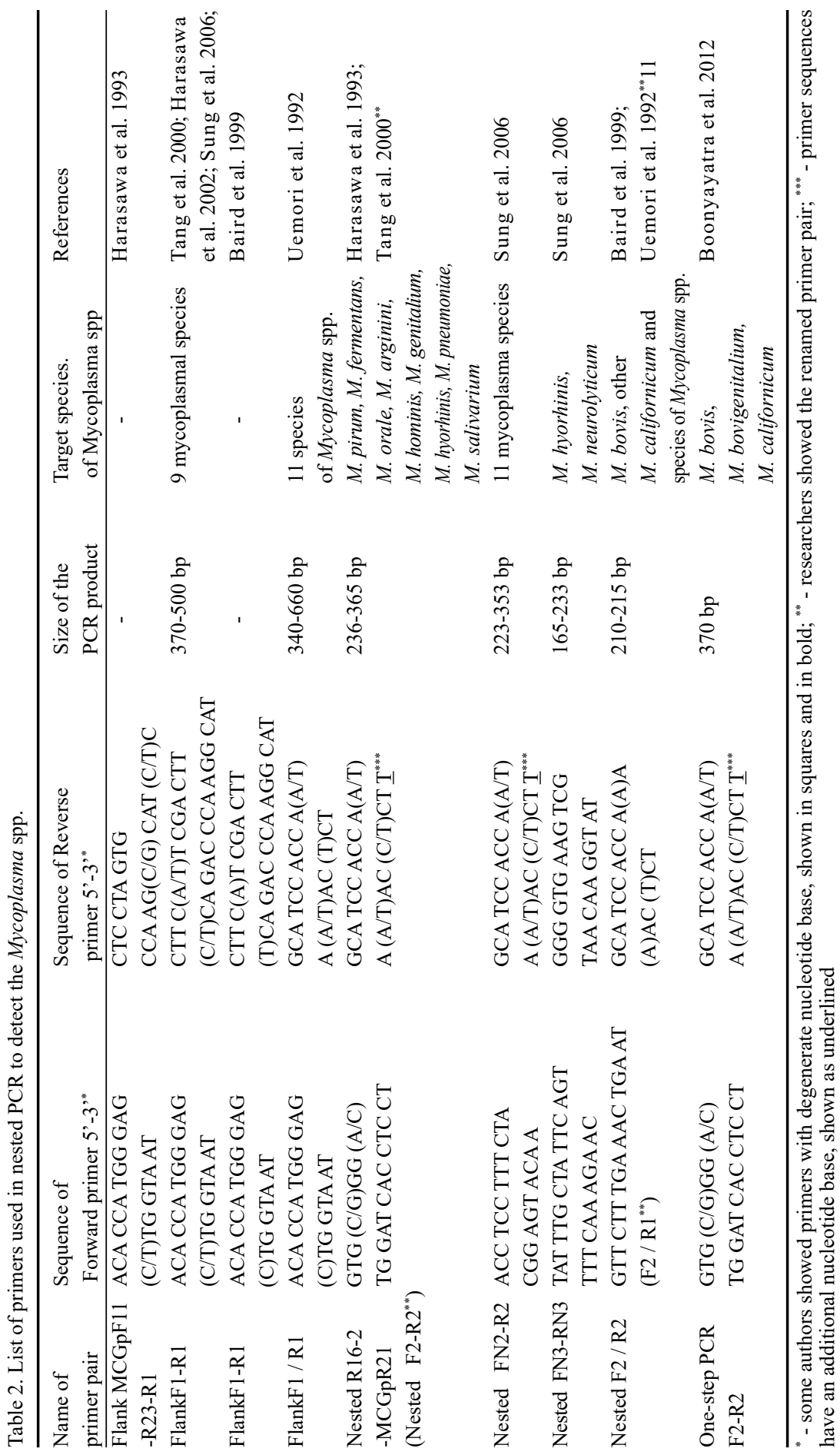


et al. (2002). Except for research by Tang et al. (2000), these universal F1-R1 primers are found in studies to identify 14 serovars of Ureaplasma urealyticum (Harasawa and Kanamoto 1999). For the first time, these oligonucleotides F1 and R2, homologous to the corresponding genes of $16 \mathrm{~S}$ and $23 \mathrm{~S}$ rRNA, were developed and used in the first round of PCR and denoted as F1 and R1 (Uemori et al. 1992). In the nested PCR with 8 different species of mycoplasma contaminants in the cell cultures, the results of Tang et al. (2000) showed generating a single DNA fragment in the range of 236-365 base pairs (bp), while A. laylawii produced a characteristic two-banded pattern with amplicons of 426 and $219 \mathrm{bp}$. The researcher carried out further species identification of mycoplasmas using endonucleases of $V s p \mathrm{I}, C l a \mathrm{I}$ and/or HindIII to cleave the obtained PCR products. However, some of the PCR results of Tang et al. (2000) and 80 et al. (1993) do not coincide while using the same restriction endonucleases and studying the same Mycoplasma spp. It is clear that these discrepancies are related to the use of different pairs of oligonucleotides in PCR.

For the purpose of PCR discrimination of different Mycoplasma spp., primers are being designed from sequences not only conserved regions of the $16 \mathrm{~S} r \mathrm{RNA}$ gene and variable $16 \mathrm{~S}-23 \mathrm{~S}$ rRNA spacer region, but researchers have also utilized primers specific to other targets (Harasawa et al. 2004). Although many researchers used a two-stage PCR method developed by Harasawa et al. (1993) with the above-mentioned primer pairs and various modifications to detect the Mycoplasma. Initially, Harasawa et al. (1993) and Tang et al. (2000) optimized the PCR method to differentiate 9 mycoplasma species of $M$. pirum, $M$. fermentans, $M$. orale, $M$. arginini, $M$. hominis, $M$. genitalium, $M$. hyorhinis, M. pneumoniae, $M$. salivarium, and A. laylawii as the most common contaminants of cell cultures. Sung et al. (2006) used the nested-PCR with F1-R1 primers for the first round to detect 13 species of mycoplasmal contaminants, including the above mentioned species, and also $M$. arthritidis, $M$. bovis, . neurolyticum, $M$. pulmonis. However, after the first stage of PCR, DNA fragments were not found for all species of Mycoplasma. Therefore, the researchers had to use R2 and designed FN2 primers in the second round of PCR for 11 species of Mycoplasma, and another internal pair FN3-RN3 for the species of M. hyorhinis and $M$. neurolyticum. Accurate species specific PCR identification of mycoplasmas may require up to three or more pairs of oligonucleotides for each species (Kong et al. 2001).

Boonyayatra et al. (2012) developed new real-time PCR assays for detecting three mycoplasma species causing cattle mastitis, and the obtained results were compared using 16S rRNA partial sequencing. These researchers also used a single-stage PCR described earlier by Tang et al. (2000) with the previously mentioned F2-R2 primers, the published sequences of which coincide, as can be seen in Table 2 (Nakagawa et al. 1992). As a result, PCR products for type strains of Mycoplasma spp. were $370 \mathrm{bp}$, that were processed by enzyme Ase 1 having the same restriction site of $\mathrm{AT}^{\wedge} \mathrm{TAAT}$ as well as for $V s p$ I. After the restriction, digested fragments 140, 125 and 115 bp were produced for M. bovis, 265 and $115 \mathrm{bp}$ are for M. bovigenitalium, while the DNA amplicon of $M$. californicum could not be cut by this enzyme. Species identified by $16 \mathrm{~S}$ rRNA sequence analysis were confirmed in 100\% agreement by PCR-RFLP and real time PCR methods for all investigated 228 of $M$. bovis and 22 of $M$. californicum field isolates. However, none of 5 field isolates of $M$. bovigenitalium were correctly identified, since the restriction profile of two strains was similar to those of $M$. californicum, and the other three isolates had quite different DNA pattern (Boonyayatra et al. 2012).

In a nested PCR reaction consisting of two rounds of amplification, Baird et al. (1999) with reference to Uemori et al. (1992) used the same commercially available outer F1 and R1 primers as well as internal F2 and R2 primers. It is indicated that flanking primers $\mathrm{F} 1$ and R1 are designed to amplify the $16 \mathrm{~S}-23 \mathrm{~S}$ rRNA spacer region, while internal F2 and R2 are annealed at the intergenic 16S-23S rRNA sequence and at the 3'-end of the 23S rRNA gene, respectively. From Table 2 it can be seen that the nucleotide sequence of 
forward F2 primer differs from the sequences presented in the published researches of Tang et al. (2000) and Boonyayatra et al. (2012). Baird et al. (1999) used F1, F2, and R2 primers in PCR, the sequences of which are presented in the study by Uemori et al. (1992) but were labelled as F1, F2, and R1, respectively. Due to DNA amplification in the first and second stages of nested PCR, fragments with different lengths were produced for mycoplasmas $M$. bovis, $M$. alkalescens, $M$. californicum, M. bovigenitalium and $M$. canadense. Two-stage PCR using these primers made it possible to differentiate the two species of $M$. bovis and $M$. californicum, which had unique amplicons of 210 and $215 \mathrm{bp}$, respectively. The standard PCR was used by other researchers to quickly and easily screen mycoplasma mastitis in dairy cattle, and to develop a simplified version of PCR detection procedure that excludes the DNA isolation stage (Higuchi et al. 2011).

\section{Oligonucleotides specificity for PCR detection}

Above is described the use of the PCR technique for the detection of bovine mycoplasmas along with the primer pairs originally designed to identify the mycoplasmal species contaminating animal cell cultures. The international nucleotide sequence databases for microorganisms are being updated, which makes it possible to check the specificity of previously designed primers in relation to different mycoplasma genomes (https: //www.ncbi.nlm.nih.gov/nuccore/). We used the commonly known PrimerBlast program to check the Flank F1-R1 primers specificity for five selected organisms of Mycoplasma spp. Genome sequences of $M$. bovis PG45 (NC 014760.1), M. californicum strain ST-6 (NZ_CP007521.1), M. bovigenitalium strain NCTC10122 (NZ_LR214970.1), M. canadense strain HAZ360 (NZ_AP014631.1) and M. bovirhinis strain NCTC10118 (NZ_LR214972.1) are available in ĞenBank database.

In comparison to M. canadense strain HAZ360 and M. bovirhinis strain NCTC10118, the sequences of two loci in M. bovis PG45 and M. bovigenitalium strain NCTC10122 do not have perfect matches to the F1-oligonucleotide, due to the difference in one nucleotide (Fig. 1, underlined nucleotide A). Whereas one of the two locus sequences of M. californicum ST-6 have two mismatches (underlined GA) to this primer on the 5'-end. The targeted sequences of 5 mycoplasma species have near perfect matches to the reverse R1-primer because of differences concerning the degenerate bases already represented in this oligonucleotide sequence (Figs 1 and 2). In PCR with the F1-R1 primers, it is assumed that the sizes of amplicons for these selected targets were ranging between 478 and $490 \mathrm{bp}$, except for M. canadense HAZ360 (367 bp).

\begin{tabular}{|c|c|c|c|c|c|c|c|c|c|c|c|c|c|c|c|c|c|c|c|c|c|c|}
\hline Flank F1 $5^{\prime} \rightarrow 3^{\prime}$ & & A & & A & & C & & & $c$ & $\mathrm{G}$ & G & A & & $\mathbf{G}$ & $\mathrm{CT}$ & $\mathbf{T}$ & G & G & $\mathbf{T}$ & A & A & $\mathrm{T}$ \\
\hline $\begin{array}{l}\text { NZ_LR214972.1.Mycoplasma } \\
\text { bowirhinis strain NCTC10118 }\end{array}$ & $822815-822834$ & . & . & - & . & . & . & . & . & . & . & 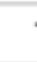 & & . & C & . & . & . & . & . & . & 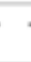 \\
\hline$\|\sqrt{ }\|$ & $694584-694603$ & . & . & . & . & . & . & . & . & . & . & 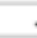 & 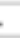 & . & C & . & . & . & . & . & . & . \\
\hline$\| \sqrt{ } / I$ & 611534.611553 & . & . & . & . & . & , & . & . & . & . & . & 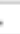 & . & C & . & . & . & . & , & • & . \\
\hline $\begin{array}{l}\text { NZ_AP014631.1 Mycoplasma } \\
\text { canadense strain HAZ360_1 }\end{array}$ & 605663.605682 & . & . & - & • & - & - & . & . & . & • & . & * & . & C & • & • & • & . & • & . & 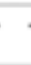 \\
\hline$\|/\|$ & $331959-331978$ & , & , & - & • & . & , & 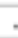 & 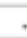 & . & • & . & • & . & C & . & . & . & . & , & . & 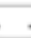 \\
\hline $\begin{array}{l}\text { NZ_LR214970.1 Mycoplasma } \\
\text { bovigenitalium strain }\end{array}$ & $256316-256335$ & . & $\Delta$ & . & • & . & . & . & . & . & . & & . & . & C & . & . & . & . & . & . & 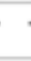 \\
\hline$\|+\|$ & $567991-567972$ & . & $\mathbf{A}$ & . & & . & . & . & . & . & - & & . & . & C & . & . & . & - & . & . & 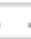 \\
\hline $\begin{array}{l}\text { NZ_CP007521.1 Mycoplasma } \\
\text { califormicum strain ST-6 }\end{array}$ & $595800-595781$ & . & $\Delta$ & - & - & . & = & . & . & . & . & & * & . & C & - & . & . & - & . & . & , \\
\hline$\|\cdot\|$ & $555814-555795$ & G & A & - & 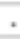 & . & . & 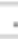 & 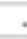 & . & 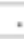 & & & . & C & . & * & 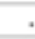 & . & . & . & \\
\hline $\begin{array}{l}\text { NC_014760.1 Mycoplasma bovis } \\
\text { PG45 }\end{array}$ & $322481-322500$ & . & $\mathbf{A}$ & * & * & . & * & " & 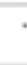 & . & * & & & & C & $\cdot$ & 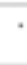 & 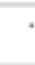 & . & . & . & \\
\hline$\| \sqrt{ } / I$ & $317487-317506$ & * & A & * & & ${ }^{\circ}$ & - & & & & & & & . & C & & & & . & . & . & \\
\hline
\end{tabular}

Fig 1. Flank F1 primer specificity to selected Mycoplasma spp. sequences 
Dlakk RI $5^{\prime} \rightarrow 3^{*}$

NZ_LR214972.1 Mocoplasma Bovirkinis sorain NCTC10118

WQII

WQII

NZ AFot $\$ 631.1$ Mycoplasma casadese strain ILAZ360 I

UVII

NZ LR214970.1 Moroplaima bontgernitalihum strain

WVeli

NZ_CP007521.1 Myroptasma californicam atrain ST-6

UVAll 555329.555355

NC_014760.1 Mpooplacma Bovis 322965-322942 Pats

"IVII

317976.317950
C T T C NT T C GACT T CT C A GAC C A A G G C A T

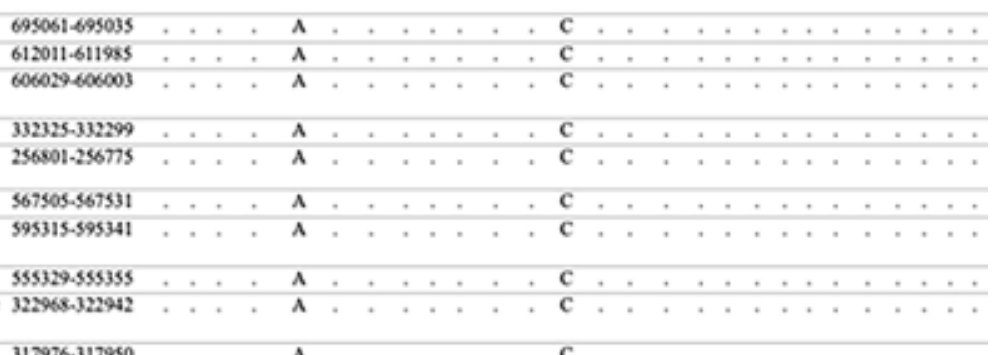

Fig 2. Flank R1 primer specificity to selected Mycoplasma spp. sequences

Nested F2 $5^{\prime \prime}>3^{*}$

NZ_AP014631.1 Mycoplasma canadense strain HAZ360 I

NZ_LR214970.1 Mycoplasma

bovigenitalium strain

II

NZ CP007521.1 M/jcoplasma

califormicum strain ST.6

$\|+\|$

NC_014760.1 Mfeoplasma

bovis PG45

$\|4\|$

NZ_LR214972.1 Mbroplasma

bovirhinis strain NCTC10118

$\|A\|$

$\| \sqrt{\|}$

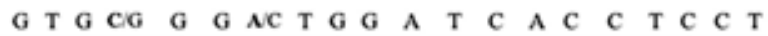

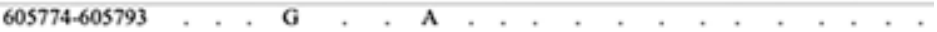
$332070-332089$

$256419-256438$ \$67888-\$67869 $\$ 55711-\$ 55692$

595698-595679 322584-322603

317590-317609 $822927-822946$

694696-694715 $611646 \cdot 611665$

$G$

+
+
+

$+$.

$G$
$G$

.$+\mathrm{G}$
.$+\mathrm{G}$

- A A .

.,$+ G+$
.,$+ G$.

A,-1

$+. \quad G$

Fig 3. Nested F2 primer specificity to selected Mycoplasma spp. sequences

\section{Nested R2 $5^{*} \rightarrow 3^{*}$}

NZ AP014631.1 Mycoplasma canadense strain HAZ360_

NZ_LR214970.1 M/ycoplasma

bovigenitalium strain

$\|+\|$

NZ CP007\$21.1 Mycoplasma

califormicum strain ST.6

NC_014760.1 Mycoplasma bovis PG45 Uन//
NZ_LR214972.1 Mycoplasma
bovirhinis strain NCTC10118

$\|+\|$

$\|=\|$
G C A T C C A A C C A AT A AT A C C T C $606006-605988$

\begin{tabular}{|c|c|c|c|c|c|c|c|c|c|c|c|c|c|c|c|c|c|c|c|}
\hline $332302-332284$ & * & . & . & - & . & " & . & . & . & - & A & . & A & . & $\cdot$ & $\mathbf{T}$ & . & . & . \\
\hline 256778.256760 & * & . & . & . & . & - & . & - & . & . & C & . & A & . & $\cdot$ & $\mathbf{T}$ & . & . & . \\
\hline 567528.567546 & . & . & . & * & . & * & . & • & . & . & C & . & A & . & • & $\mathbf{T}$ & . & $\cdot$ & * \\
\hline$\$ 55352-555370$ & * & . & . & * & . & * & * & - & • & . & C & . & $A$ & . & $\theta^{\circ}$ & $\mathbf{T}$ & . & * & . \\
\hline 595338.595356 & * & . & . & ${ }^{\circ}$ & . & " & * & - & . & • & C & . & $A$ & . & • & $\mathbf{T}$ & . & ${ }^{*}$ & * \\
\hline $322945-322927$ & * & * & . & * & . & * & * & • & $\cdot$ & • & C & $\cdot$ & A & . & $\cdot$ & $\mathbf{T}$ & - & * & . \\
\hline 317953.317935 & $*$ & - & - & . & * & * & . & ${ }^{*}$ & $\cdot$ & ${ }^{*}$ & C & $\cdot$ & $\mathrm{A}$ & . & $\cdot$ & $\mathbf{T}$ & . & 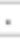 & * \\
\hline $823269-823251$ & * & * & • & $\cdot$ & - & - & * & • & $\cdot$ & . & C & $\mathrm{T}$ & C & $\cdot$ & - & $\mathrm{T}$ & . & * & * \\
\hline $695038-695020$ & * & * & . & . & . & * & - & $\cdot$ & . & $*$ & C & $\mathrm{I}$ & $\mathrm{C}$ & . & - & $\mathbf{T}$ & . & ${ }^{*}$ & 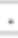 \\
\hline $611988-611970$ & • & * & * & - & * & & , & & - & & C & $\mathrm{I}$ & C & . & - & $\mathbf{T}$ & . & • & \\
\hline
\end{tabular}

Fig 4. Nested R2 primer specificity to selected sequences of Mycoplasma spp. 
Analyzing the nested F2-R2 primer specificity, it was found that the sequences of the investigated mycoplasma genomes have one mismatch to the F2-primer sequence excluding the almost fully matched sequence of $M$. canadense HAZ360. As shown in Fig. 3, one of the two studied loci of M. californicum ST-6 has 2 nucleotides mismatched to the F2-primer. The reverse R2 primer is not fully specific to the sequences of 2 loci in $M$. bovis PG45, M. bovigenitalium NCTC10122, and M. californicum ST-6, having one mismatch. It is interesting that sequences located in three genetic loci of $M$. bovirhinis NCTC10118 have significant number of mismatches (3 nucleotides) to the R2 sequence (Fig. 4).

It is predicted that nested PCR with F2-R2 and targets of $M$. bovirhinis NCTC10118, M. bovigenitalium NCTC10122, M. californicum ST-6 and M. bovis PG45 can produce fragments with 343-364 bp in size, and 233 bp amplicon is for M. canadense HAZ360. In general, the PrimerBlast allowed to check the flank F1-R1 and nested F2-R2 primer pairs specificity to 83 and 82 of RefSeq representative genomes. The specificity of F2/R2 primers using by Baird S.C. in second-stage of nested PCR was also checked but against to 170 genome sequences of Mycoplasma spp. It was found that these primers have 2-5 mismatches to different loci of many genome sequences available through the GenBank database.

\section{Mycoplasma species specific primers}

The computer alignment of the known 16S rRNA gene sequences for 36 species and mycoplasma strains, revealed some consistent differences in the V2 and V3 regions of the gene. Based on these differences in 16S rRNA nucleotide sequences, species-specific primers have been selected for 8 species of Mycoplasma spp. and also for U. urealyticum isolated from human biomaterials and rats (Van Kuppeveld et al. 1992). Three speciesspecific pairs of primers have been developed using the pMB2, pMB900, pMB920, pJ5 plasmid library containing inserts of the M. bovis genome (Hotzel et al. 1993). It was shown that PCR with M. bovis-specific PpMB2 primers do not cross-react with DNA samples of 27 other mycoplasmal species. However, the PpMB920 F/R primers along with the previously designed oligonucleotides for $M$. alkalescens, M. bovigenitalium, and $M$. bovirhinis associated with mastitis and mycoplasma pneumonia in cattle were used by Hirose et al. (2001) to detect the M. bovis species. By using species-specific PCR, $M$. bovirhinis was detected in $70 \%$ of milk samples from cows with clinical mastitis symptoms whereas $M$. bovis found only in $7 \%$ of them.

For mycoplasma detection, Brazilian researchers used previously developed primers species-specific to the $16 \mathrm{~S}$ rRNA gene of $M$. bovigenitalium and $U$. urealyticum (Masedo et al. 2018). These oligonucleotides were used in a multiplex PCR detection of M. bovis, $M$. bovigenitalium, and $U$. urealyticum species in the same biological sample (Tramuta et al. 2011). Three pairs of primers were used to detect the three mycoplasmal species and as can be seen in Table 3, the sequences of the forward primer in these pairs were identical.

\section{Real time PCR for mycoplasma detection}

Among the molecular typing techniques, the multilocus variable number of tandem repeats analysis (MLVA) method is new and used to characterise and differentiate the field and vaccine strains of $M$. synoviae, as an important poultry pathogen (Kreizinger et al. 2018). The same approach with evaluated multilocus sequence typing (MLST) showed the highest resolution power to differentiate 40 strains of 44 genotypes of $M$. hyopneumoniae, a common swine pathogen (Feldea et al. 2018). However, the multilocus sequencing analysis based on identifying up to 302 nuclear genes of the $M$. synoviae genome, is very 


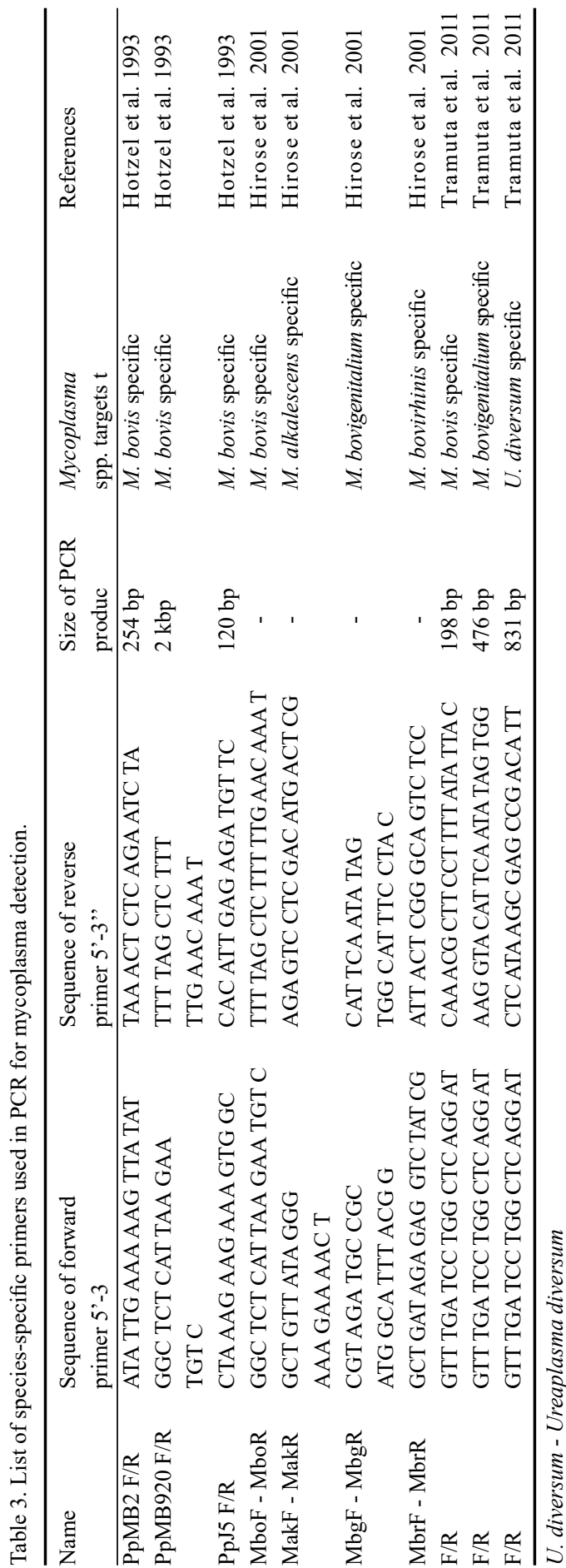

expensive and therefore not used in routine diagnostics for mycoplasma detection (Ghanem and El-Gazzar 2018).

In over than the last decade, the real-time PCR method based on using hybridization probes has been introduced into clinical microbiology. This technique allows not only to detect the presence of mycoplasmas in mastitis milk samples and animal tissues in real time within a few hours, but also to differentiate between the closely related mycoplasma species (Cai et al. 2005). To detect M. bovis in the milk and lung tissue of cattle, Cai et al. (2005) developed the real-time PCR method with MbsF and MbsR primers and a pair of FRET probes, the sequences of which are presented in Table 4. Selection of MbsF and MbsR oligonucleotides was based on its specificity to the $16 \mathrm{~S}$ rRNA genes of infectious mastitis pathogens including M. bovis, M. agalactiae, Staphylococcus aureus and Streptococcus agalactiae.

The DNA probe set contained at least 2 nucleotides mismatches to the sequence of the M. agalactiae $16 \mathrm{~S}$ rRNA gene and was therefore used to detect differences in melting temperatures of hybridized probe and the PCR fragment of $M$. bovis or $M$. agalactiae. Of all the tested bovine mycoplasma and other bacteria, only $M$. bovis strains were positive in the real time $\mathrm{PCR}$, with a melting peak of $66.6^{\circ} \mathrm{C}$ for the DNA fragments, and a positive result was obtained with $M$. agalactiae strain PG2 having a lower melting peak of $63.1^{\circ} \mathrm{C}$.

The protocol utilizing cyanine SYBR-green dye was found to be a less expensive version for the real time PCR technique, which does not require labelled DNA-probes. Harasawa et al. (2005) used a realtime-SYBR-PCR with F1-R1 primers developed previously for conventional PCR to detect 5 mycoplasma species contaminating the cell lines. This analysis showed that mycoplasma 


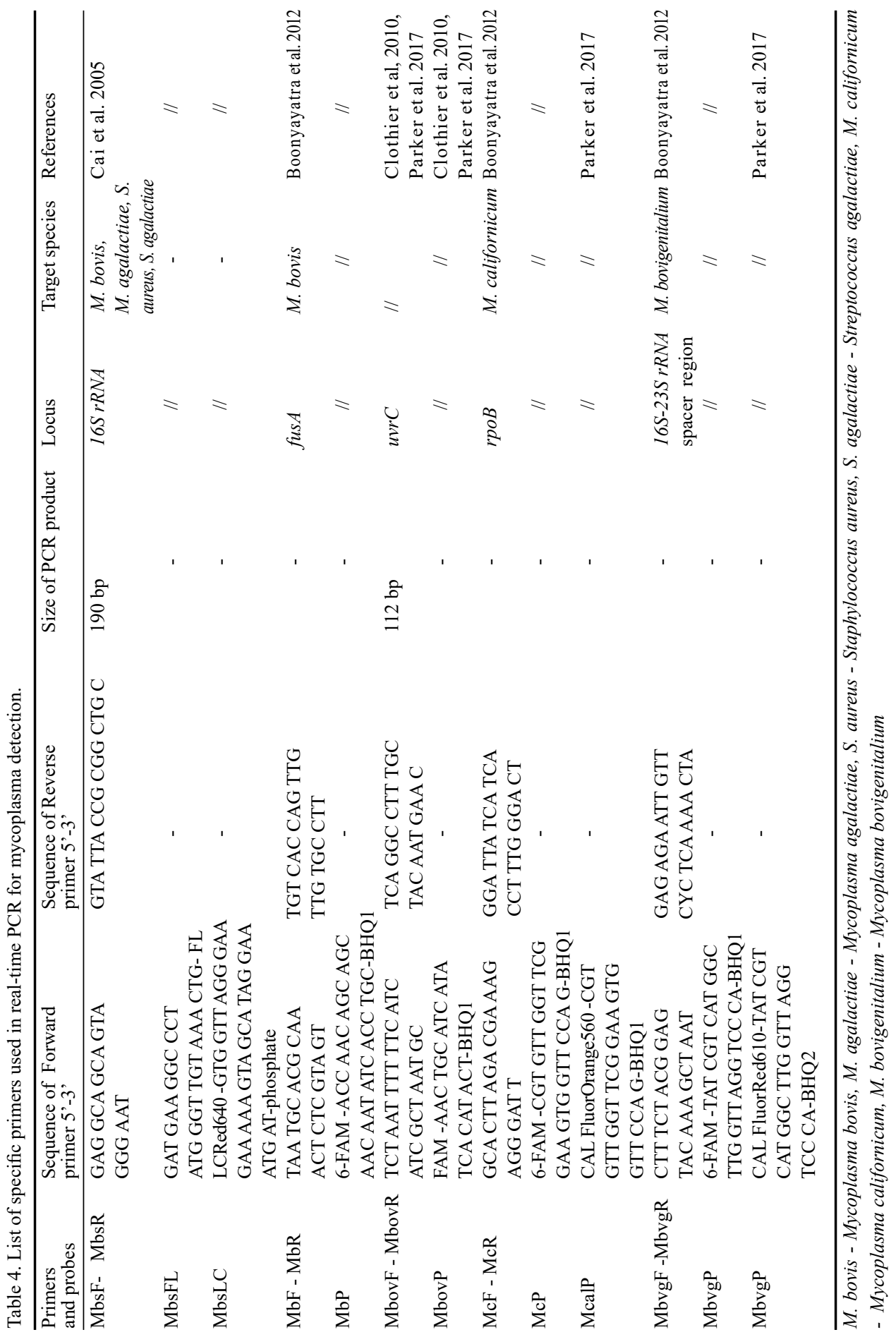


strains have different melting temperatures $(T \mathrm{~m})$ of double-stranded DNA in respect with Mycoplasma species. The values of $T \mathrm{~m}$ were $89.14-89.54{ }^{\circ} \mathrm{C}$ for $M$. orale, $89.02-89.42^{\circ} \mathrm{C}$ for M. fermentans, $88.54-88.94{ }^{\circ} \mathrm{C}$ for M. hyorhinis, $87.68-$ $87.98{ }^{\circ} \mathrm{C}$ for $M$. arginini, and $87.25-87.65^{\circ} \mathrm{C}$ for $M$. salivarium.

By Justice-Allen et al. (2011), the SYBR-PCR method was used to detect the presence of and to identify several mycoplasma species other than M. bovis. The researchers reported using primers that were previously developed and specific to the conserved locus of rRNA intergenic region of the Mycoplasma genus. In addition to this technique, PCR amplicon sequencing was used to confirm species identity in cases where the melting temperature of the DNA fragment did not correspond to the typical $T \mathrm{~m}$ value of $73.2{ }^{\circ} \mathrm{C}$ for the $M$. bovis species. The results of this study showed that milk samples from a bulk tank with a negative result of microbiological assessment had a positive test for the presence of M. bovigenitalium in the SYBR-PCR assays. So this culture negative sample was identified as $M$. bovigenitalium positive and in PCR analysis produced the amplicon with size of 222 bp and $T \mathrm{~m} 73.18^{\circ} \mathrm{C}$. The PCR M. bovis-positive samples had an amplified product of $207 \mathrm{bp}$ and $\mathrm{Tm}=73.39{ }^{\circ} \mathrm{C}$, compared to mycoplasmas other that $M$. bovis tended to have lower Tm and plus a different PCR product in length (approximately $156 \mathrm{bp}$ ). In comparison to the traditional microbiological method, the SYBR-PCR analysis did not show any significant sensitivity when detecting different species of Mycoplasma spp. in bulk tank milk.

In order to avoid cross-amplification and to precisely identify the closely related mycoplasma species including $M$. bovis and $M$. agalactiae, the researchers recommend to use designed primers specific to different genetic loci of genome DNA isolating from complex biomaterials. Thus Boonyayatra et al. (2012) developed 3 assays of real-time PCR with primer pairs and FAM-labelled DNA probes specific to different loci of the three mastitis agents $M$. bovis, M. californicum, and M. bovigenitalium.

Table 4 presents sequences of the primer pairs Mb-F and Mb-R, McF and McR, Mbvg-F and Mbvg-R that are specific to the fusA gene (encoding for elongation factor $\mathrm{G}$ ) of $M$. bovis, the gene rpoB (encoding for RNA polymerase $\beta$ subunit) of $M$. californicum, and the 16S-23S rRNA spacer region of $M$. bovigenitalium, respectively. The results of the mycoplasma detection were fully consistent with the 16S rRNA gene sequencing analysis, except for 4 samples identified by real time PCR as M. bovis and M. californicum. It is known that the method of $16 \mathrm{~S}$ rRNA partial sequencing allows detecting a dominant species of microorganisms, therefore the sequencing of all the produced amplicons can confirm the presence of two mycoplasma species in samples which was done by the authors for these cases.

Moreover, a multiplex real-time PCR with different species-specific DNA probes labelled with FAM, CAL Fluor Orange 560 and CAL Fluor Red 610 has been developed in order to detect these three mycoplasma species in bovine milk, semen and swab samples (Parker et al. 2017). In their research for rt-PCR identification of $M$. bovigenitalium and $M$. californicum, Parker et al. (2017) applied DNA probes and primers MbvgF and MbvgR, McalF and McalR described by Boonyayatra et al. (2012) and also MbovP, MbovF and MbovR oligonucleotides specific to $M$. bovis $u v r C$ gene and developed by Clothier et al. (2010).

By using the mentioned DNA extraction methods, the multiplex rt-PCR detection limits for mycoplasma $M$. bovis and $M$. californicum in milk samples were $1.3 \times 10^{2} \mathrm{CFU} / \mathrm{ml}$ and $6.0 \times 10^{2} \mathrm{CFU} / \mathrm{ml}$ respectively. According to Clothier et al. (2010), the detection level of $M$. bovis in all spikes of milk, lung tissue and suspension culture samples was $2.4 \times 10^{2}$ $\mathrm{CFU} / \mathrm{ml}$ whereas 10-30 CFU/ml were reported by Boonyayatra et al. (2012) in the single rt-PCR assay. Although the newly developed multiplex real-time PCR had a poorer limit for $M$. bovigenitalium detection in contaminated milk $\left(5.0 \times 10^{5} \mathrm{CFU} / \mathrm{ml}\right)$ compared to other mycoplasma species (Parker et al. 2017). 


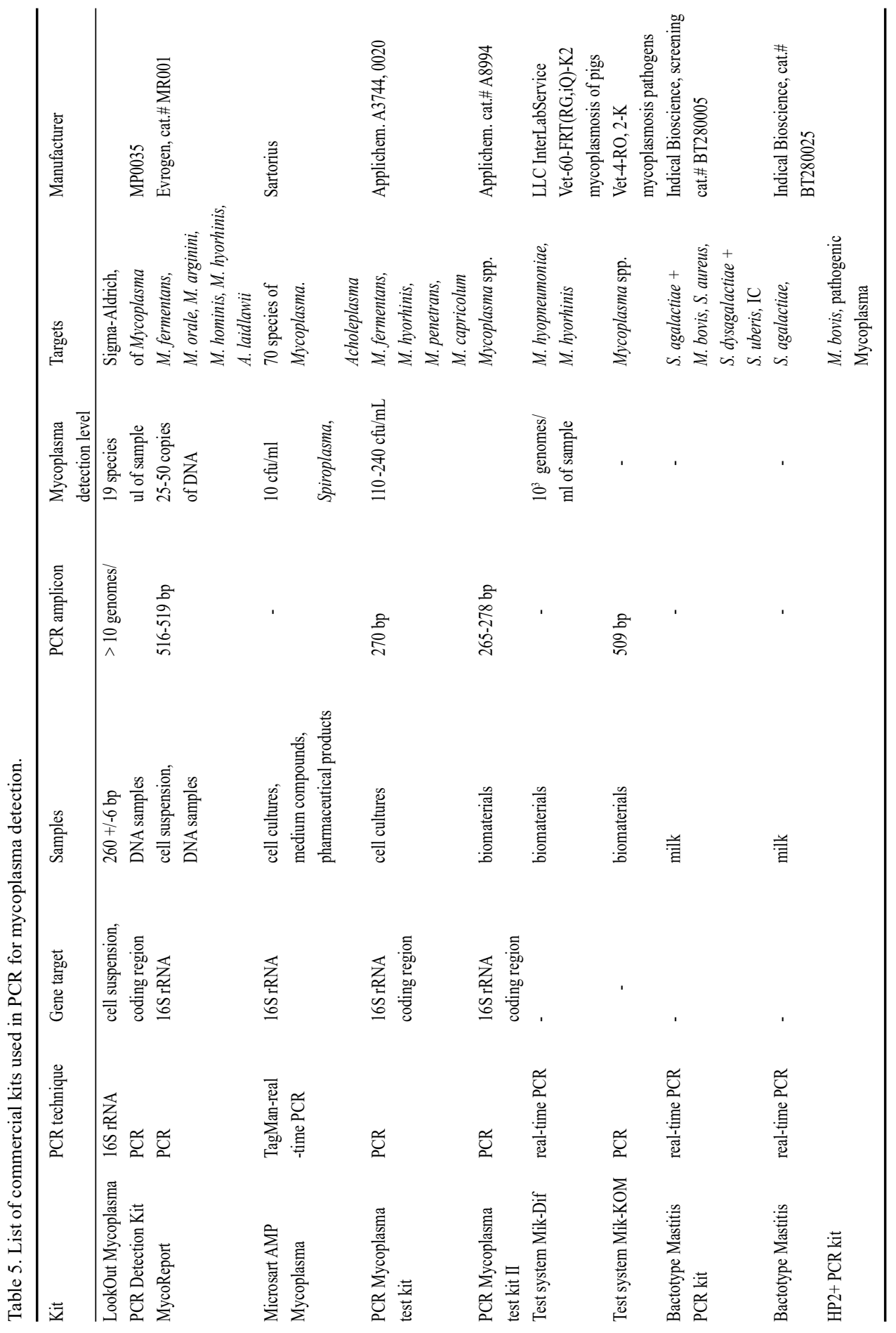




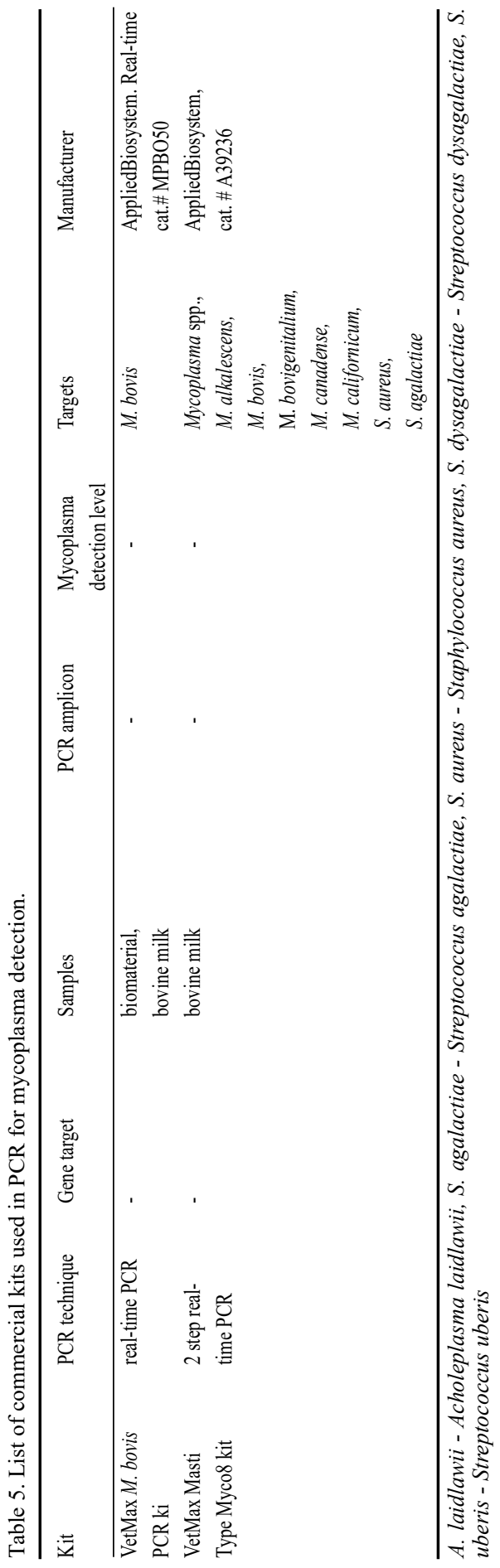

\section{PCR detection kits for Mycoplasma spp.}

Most Russian companies offer PCR kits to detect the mycoplasmas belonging to human pathogens, and also mycoplasma contaminants in cell line cultures (Table 5). The MycoReport and Myco real-time PCR assay kits for detecting mycoplasma contaminants in cell cultures are commercially available from the Eurogen company. The PCR test systems developed by the Federal Budget Institution of Science "The Central Research Institute of Epidemiology" (FBIS CRIE) are offered throughLLCInterLabService and used to detect the swine pathogens of $M$. hyopneumoniae, $M$. hyorhinis, the poultry pathogens M. gallisepticum, M. synoviae, and include the Mik-Kom test system for detecting various mycoplasma species.

Numerous foreign manufacturers such as Norgen Biotek Corp. (Canada), PromoCell and Sartorius (Germany) also offer mainly PCR detection systems for mycoplasma in cell culture and for use in research only and not for diagnostics. The MycoSEQ Mycoplasma real-time PCR system based on SYBR green technology for detecting 90 different mycoplasma species, acholeplasma and spiroplasma as contaminants of cell cultures and nutrient media, was developed for use in research only by Applied Biosystems. The AB company developed the VetMax M. bovis realtime PCR kit to $M$. bovis diagnosis in various samples, including milk, as well as the VetMax MastiType Multi Kit, MastiType Myco8 Kit, MastiType Micro4 Kit for the detection of mastitis pathogens. Sigma-Aldrich (USA) offers the LookOut Mycoplasma PCR Detection Kit, which can be used for research and in diagnostic procedures to detect mycoplasmal contamination of cell cultures. This kit contains 8-strip microtubes that are pre-coated with dNTPs and mycoplasmaspecific primers and also DNA-positive tubes as an internal control (Dobrovolny and Bess 2011). For veterinary use only, the assays with qPCR detection of Streptococcus agalactiae, M. bovis, Staphylococcus aureus, Streptococcus dysagalactiae, and Streptococcus uberis are available from the Indical Bioscience (Germany), formerly Qiagen Leipzig. 


\section{Conclusions}

Among more than 100 species of Mycoplasma, many species are recognized as common contaminants in cell cultures while others are of interest in cattle. Mycoplasma species are associated with a variety of clinical diseases such as mastitis, arthritis, pneumonia, vulvovaginitis, endometritis and cause the abortion and infertility in infected herds. Outbreaks of the most important mycoplasmas can lead to serious economic losses in farm animals worldwide.

Microbiological culture is an available standard method for routine testing of mycoplasmas and should be followed by serological analysis. For the definitive species identification, one of these techniques is laborious and requires up to a month for mycoplasma growth on several appropriate culture media; the second test can be used with a species-specific antibody and only on clinical animals. At the present time, using PCR technique for mycoplasma detection is becoming more common because it allows to obtain diagnostic results quickly so that the cows shedding pathogens can be effectively removed from the herd at an early stage of infection.

Different PCR protocols for mycoplasma detection have been developed over the last three decades. This process is ongoing with collecting new data on the disease pathogenesis and strains of mycoplasma agents (Gondaira et al. 2017). Compared to methods of gene sequencing, multilocus typing and real-time PCR, traditional and multiplex PCR methods are the simplest and less expensive (Higuchi et al. 2011). It is known that the efficiency of PCR detection of microorganisms largely depends on the level of contamination of the biological sample and the type of material under study. Due to the low initial level of the infection agent, particularly in subclinical animals, many researchers have to optimize the PCR protocol to directly detect mycoplasmas in various materials, including milk, lung tissue, excretion, swabs (Rossetti et al. 2010; Justice-Allen et al. 2011; Parker et al. 2017).

In order to avoid cross-amplification and to precisely identify the closely related mycoplasma species, the researchers recommend to use designed primers specific to different genetic loci of the mycoplasmal genome. So, according to peer-reviewed reports, we can see the use of the PCR technique for the detection of bovine mycoplasmas along with the primer pairs originally designed to identify the mycoplasma species contaminating animal cell cultures. Therefore, it was interesting to verify the specificity of some of these primers to available genome sequences of five Mycoplasma spp., as clinically important agents in cattle especially in the international nucleotide sequences databases for microorganisms are always being updated. We have to notice an interesting finding given that the F1-R1 and F2-R2 primer pairs recommended by the researchers for the nested-PCR were specific to $M$. bovirhinis NCTC10118 sequences located in three genetic loci (Baird et al. 1999; Tang et al. 2000; Harasawa et al. 2002; Sung et al. 2006). This case may be explained by a low primer specificity for the target because it is known that mycoplasmas have 1 or $2 r$ RNA operons, although the genome of $M$. bovirhinis species is still not very much established (Nakagawa et al. 1992).

The commercially available PCR mycoplasma detection kits have been developed based on the approaches revised in this study. However, it is important to develop a new multiplex PCR assay for the purpose of identifying Mycoplasma spp. other than M. bovis on animal farms with mastitis outbreaks as reported by some researchers. Along with this, a new multiplex PCR protocol should be adapted to accurately identify all species without depending on a concentration of them in complex type samples (Parker et al. 2017).

Government programs are aimed to support the development of livestock industry in Kazakhstan but breeders have problems with decreased cow fertility and abortion along with the breeding of highly valuable and productive animals. As showed in the literature, the said problems are widely encountered on large farms using artificial insemination and having 
animals transported from foreign countries. Antimicrobial therapy for mycoplasmosis is not effective because of mycoplasma mutations responsible for resistance to a wide range of drugs (Sulyok et al. 2018).

In Kazakhstan, no studies have been conducted on the most prevalent and clinically important mycoplasma species in cattle and no data on the circulating mycoplasma strains are available. In general, the practical importance of PCR methods for mycoplasma detection lies in early diagnosis of the pathogens and providing effective veterinary control, which is especially important in countries with a predominance of livestock industry.

\section{Acknowledgements}

The study was supported by the Ministry of Agriculture of the Republic of Kazakhstan and the Kazakh Scientific Research Veterinary Institute as part of targeted funding for the program "Scientific support for veterinary welfare and food safety" $267 / 101 / 156$.

\section{References}

Adilbekova AK, Usenbekov ES, Turebekov OT, Koibagarov KU, Mahmutov AK 2015: Physiotherapeutic methods for treating mastitis in cows (in Russian). Intellect Idea Innovation 1: 19-24

Baird SC, Carman J, Dinsmore RP, Walker RL, Collins JK 1999: Detection and identification of Mycoplasma from bovine mastitis infections using a nested polymerase chain reaction. J Vet Diagn Invest 11: 432-435

Boonyayatra S, Fox LK, Besser TE, Sawant A, Gay JM, Raviv Z 2012: A PCR assay and PCR-restriction fragment length polymorphism combination identifying the 3 primary Mycoplasma species causing mastitis J Dairy Sci 95: 196-205

Cai HY, Bell-Rogers P, Parker L, Prescott JF 2005: Development of a real-time PCR for detection of Mycoplasma bovis in bovine milk and lung samples. J Vet Diagn Invest 17: 537-545

Clothier KA, Jordan DM, Thompson CJ, Kinyon JM, Frank TS, Strait EL 2010: Mycoplasma bovis real-time polymerase chain reaction assay validation and diagnostic performance. J Vet Diagn Invest 22: 956-60

Dobrovolny PL, Bess D 2011: Optimized PCR-based Detection of Mycoplasma. Jo VE 52: e3057

Feldea O, Kreizinger Z, Sulyoka KM, Martona S, Bányaia Kr, Korbulya K, Kissb K, Biksic I, Gyuranecz M 2018: Genotyping Mycoplasma hyopneumoniae isolates based on multi-locus sequence typing, multiple-locus variable-number tandem repeat analysis and analysing gene. Vet Microbiol 222: 85-90

Friis NF 1975: Some recommendations concerning primary isolation of Mycoplasma suipneumoniae and Mycoplasma flocculare. Nord Veterinarmed 27: 337-339

Ghanem M, El-Gazzar M 2018: Development of Mycoplasma synoviae (MS) core genome multilocus sequence typing (cgMLST) scheme. Vet Microbiol 218: 84-89

Gondaira S, Higuchia H, Nishia K, Iwano H, Nagahata H 2017: Mycoplasma bovis escapes bovine neutrophil extracellular traps. Vet Microbiol 199: 68-73

Harasawa R, Mizusawa H, Nozawa K, Nakagawa T, Asada K, Kato I 1993: Detection and tentative identification of dominant mycoplasma species in cell cultures by restriction analysis of the 16S-23S rRNA intergenic spacer regions. Res Microbiol 144: 489-493

Harasawa R 1996: Nested PCR: Application to the detection of mycoplasmas. In: Razin S, Tully JG (Eds): Molecular and diagnostic procedures in Mycoplasmology Vol. 2. Academic press, New York, pp. 75-79

Harasawa R 1999: Genetic relationships among mycoplasmas based on the 16S-23S rRNA spacer sequence. Microbiol and Immunol 43: 127-132

Harasawa R, Kanamoto Y 1999: Differentiation of two biovars of Ureaplasma urealyticum based on the 16S-23S rRNA Intergenic spacer region. J. Clinic Microbiol 37: 4135-4138

Harasawa R, Hotzel H, Sachse K 2000: Comparison of 16S-23S $r$ RNA intergenic spacer regions among strains of the Mycoplasma mycoides cluster, and reassessment of the taxonomic position of Mycoplasma sp. bovine group 7. Intern J Syst and Evol Microbiol 50: 1325-1329

Harasawa R, Kawahara M, Rikihisa Y 2002: Characteristics of the 16S-23S rRNA Intergenic Spacer Region of Mycoplasma haemomuris, previously classified as 'Haemobartonella muris'. J Vet Med Sci 64: 1161-1164

Harasawa R, Pitcher DG, Ramirez AS, Bradbury JM 2004: A putative transposase gene in the 16S-23S rRNA intergenic spacer region of Mycoplasma imitans. Microbiology 150: 1023-1029

Harasawa R, Mizusawa H, Fujii M, Yamamoto J, Mukai H, Uemori T, Asada K, Kato I 2005: Rapid detection and differentiation of the major mycoplasma contaminants in cell cultures using real-time PCR with SYBR green I and melting curve analysis. Microbiol Immunol 49: 859-863

Higuchi H, Iwano H, Kawai K, Ohta T, Obayashi T, Hirose K, Ito N, Yokota H, Tamura Y, Nagahata H 2011: A simplified PCR assay for fast and easy mycoplasma mastitis screening in dairy cattle. J Vet Sci 12: 191-193

Hirose K, Kawasaki Y, Kotani K, Tanaka A, Abiko K, Ogawa H 2001: Detection of Mycoplasma in mastitic milk by PCR analysis and culture method. J Vet Med Sci 63: 691-693 
Hotzel H, Demuth B, Sachse K, Pflitsch A, Pfutzner H 1993: Detection of Mycoplasma bovis using in vitro deoxyribonucleic acid amplification. Rev Sci Tech 12: 581-591

Justice-Allen A, Trujillo J, Goodell G, Wilson D 2011: Detection of multiple Mycoplasma species in bulk tank milk samples using real-time PCR and conventional culture and comparison of test sensitivities. J Dairy Sci 94: 3411-3419

Kong F, James G, Gordon S, Zelynski A, Gilbert GL 2001: Species-specific PCR for identification of common contaminant mollicutes in cell culture. Appl Env Microbiol 67: 3195-3200

Kreizinger Z, Sulyok KM, Bekő K, Kovács ÁB, Grózner D, Felde O, Marton S, Bányai K, Catania S, Benčina D, Gyuranecz M 2018: Genotyping Mycoplasma synoviae: Development of a multi-locus variable number of tandem-repeats analysis and comparison with current molecular typing methods. Vet Microbiol 226: 41-49

Masedo AAM, Oliveira JMB, Silva BP, Borges JM, Soares LBF, Silva GM, Santos SB, Mota RA, PinheiroJunior JW 2018: Occurrence of Mycoplasma bovigenitalium and Ureaplasma diversum in dairy cattle from to Pernambuco state, Brazil. Arq Bras Med Vet Zootec 70: 1798-1806.

Marusich AG 2017: Cattle breeding. Herd reproduction: educational and methodical manual. UO "BSAA", Horki, $64 \mathrm{p}$.

Nakagawa T, Uemori T, Asada K, Kato I, Harasawa R 1992: Acholeplasma laidlawii has tRNA genes in the $16 \mathrm{~S}-23 \mathrm{~S}$ spacer of the rRNA operon. J Bacteriol 174: 8163-8165

Parker AM, House JK, Hazelton MS, Bosward KL, Sheehy PA 2017: Comparison of culture and a multiplex probe PCR for identifying Mycoplasma species in bovine milk, semen and swab samples. PLoS One 12: e0173422

Rossetti BC, Frey J, Pilo P 2010: Direct detection of Mycoplasma bovis in milk and tissue samples by real-time PCR. Mol Cell Probes 24: 321-323

Sachse K, Pfützner H, Hotzel H, Demuth B, Heller M, Berthold E 1993: Comparison of various diagnostic methods for the detection of Mycoplasma bovis. Rev Sci Tech 12: 571-580

Stakenborg T, Vicca J, Butaye P, Maes D, De Baere T, Verhelts R, Peeters J, De Kruif A, Haesebrouck F, Vaneechoutte M 2005: Evaluation of amplified rDNA restriction analysis (ARDRA) for the identification of Mycoplasma species. BMC Infectious diseases 5: 46

Sulyok KM, Bekő K, Kreizinger Z, Wehmann E, Jerzsele Á, Rónai Z, Turcsányi I, Makrai L, Szeredi L, Jánosi S, Nagy SA, Gyuranecz M 2018: Development of molecular methods for the rapid detection of antibiotic susceptibility of Mycoplasma bovis. Vet Microbiol 213: 47-57

Sung H, Kang SH, Bae YJ, Hong JT, Chung YB, Lee Ch-K, Song S 2006: PCR based detection of Mycoplasma species. J Microbiol 44: 42-49

Tang J, Hu M, Lee S, Roblin R 2000: A polymerase chain reaction based method for detecting Mycoplasma/ Acholeplasma contaminants in cell culture. J Microbiol Methods 39: 121-126

Tramuta C, Lacerenza D, Zoppi S, Goria M, Dondo A, Ferreglio E, Nebbia P, Rosati S 2011: Development of a set of multiplex standard polymerase chain reaction assays for the identification of infectious agents from aborted bovine clinical samples. J Vet Diagn Invest 23: 657-664

Uemori T, Asada K, Kito I, Harasawa R 1992: Amplification of the 16S-23S spacer region in rRNA operons of mycoplasmas by the polymerase chain reaction. Syst Appl Microbiol 15: 181-186

Van Kuppeveld FJM, Van Der Logt JTM, Angulo AF, Van Zoest MJ, Quint WGV, Niesters HGM, Galama JMD, Melchers WJG 1992: Genus- and species-specific identification of Mycoplasmas by 16S rRNA amplification. Appl and Environ Microbiol 58: 2606-2615

Zhumanov KT, Biyshev KB, Biyshev BK, Sansyzbai AR, Valdovska A, Oryntaev KB 2015: Comparison of some methods of diagnostics of mycosis mastitis in cows. Intellect Idea Innovation 3: 40-44 (in Russian) 